\title{
Existence results for $p$-Laplacian boundary value problems of impulsive dynamic equations on time scales
}

\section{Ozlem B Ozen, Ilkay Y Karaca* and Fatma Tokmak}

"Correspondence:

ilkay.karaca@ege.edu.tr

Department of Mathematics, Ege

University, Bornova, Izmir, 35100,

Turkey

\begin{abstract}
In this paper, Bai-Ge's fixed point theorem is used to investigate the existence of positive solutions for second-order boundary value problems of $p$-Laplacian impulsive dynamic equations on time scales. As an application, we give an example to demonstrate our results.

MSC: 34B18; 34N05; 39B37
\end{abstract}

Keywords: impulsive dynamic equation; $p$-Laplacian; positive solutions; fixed point theorems; time scales

\section{Introduction}

Impulse differential equations describe processes which experience a sudden change of state at certain moments; see the monographs of Lakshmikantham et al. [1] and Samoilenko and Perestyuk [2]. Impulsive differential equations can be used to describe a lot of natural phenomena such as the dynamics of populations subject to abrupt changes (harvesting, diseases, etc.), which cannot be described using classical differential equations. That is why in recent years they have attracted much attention of investigators ( $c f$., e.g., $[3,4])$.

The study of dynamic equations on time scales goes back to Stefan Hilger [5]. Now it is still a new area of fairly theoretical exploration in mathematics. We refer to the books by Bohner and Peterson $[6,7]$. There are a lot of works concerning the $p$-Laplacian problems on time scales; see, for example, [8-10]. Few works have been done on the existence of solutions to boundary value problems (BVP) for $p$-Laplacian impulsive dynamic equations on time scales; see [11-14]. Moreover, there is not much work on $m$-point boundary value problems for the $p$-Laplacian impulsive dynamic equations on time scales except for that in [15] by Li et al. Our aim in this paper is to fill the gap.

Motivated by the above mentioned works, in this paper we consider the existence of positive solutions of the following $m$-point boundary value problems for $p$-Laplacian im-

\section{空 Springer}

C2013 Ozen et al: licensee Springer. This is an Open Access article distributed under the terms of the Creative Commons Attribution License (http://creativecommons.org/licenses/by/2.0), which permits unrestricted use, distribution, and reproduction in any medium, provided the original work is properly cited. 
pulsive dynamic equation on time scales:

$$
\left\{\begin{array}{l}
\left(\phi_{p}\left(u^{\Delta}(t)\right)\right)^{\nabla}+q(t) f\left(t, u(t), u^{\Delta}(t)\right)=0, \quad t \in[0,1]_{\mathbb{T}}, t \neq t_{k}, k=1,2, \ldots, n, \\
\Delta u\left(t_{k}\right)=-I_{k}\left(u\left(t_{k}\right)\right), \quad k=1,2, \ldots, n, \\
\Delta \phi_{p}\left(u^{\Delta}\left(t_{k}\right)\right)=-\bar{I}_{k}\left(u\left(t_{k}\right), u^{\Delta}\left(t_{k}\right)\right), \quad k=1,2, \ldots, n, \\
\phi_{p}\left(u^{\Delta}(0)\right)=\sum_{j=1}^{m-2} \alpha_{j} \phi_{p}\left(u^{\Delta}\left(\xi_{j}\right)\right), \quad u(1)=\sum_{j=1}^{m-2} \beta_{j} u\left(\eta_{j}\right),
\end{array}\right.
$$

where $\mathbb{T}$ is a time scale, $0,1 \in \mathbb{T},[0,1]_{\mathbb{T}}=[0,1] \cap \mathbb{T}, t_{k} \in(0,1)_{\mathbb{T}}, k=1,2, \ldots, n$, with $0<$ $t_{1}<t_{2}<\cdots<t_{n}<1, \xi_{j}, \eta_{j} \in(0,1)_{\mathbb{T}}(j=1,2, \ldots, m-2)$ with $0<\xi_{1}<\xi_{2}<\cdots<\xi_{m-2}<1,0<$ $\eta_{1}<\eta_{2}<\cdots<\eta_{m-2}<1$ and $\xi_{j}, \eta_{j} \neq t_{k}, j=1,2, \ldots, m-2, k=1,2, \ldots, n$. $\phi_{p}(s)$ is a $p$-Laplacian operator, i.e., $\phi_{p}(s)=|s|^{p-2} s$ for $p>1,\left(\phi_{p}\right)^{-1}(s)=\phi_{q}(s)$, where $\frac{1}{p}+\frac{1}{q}=1$ and $\Delta u\left(t_{k}\right)=u\left(t_{k}^{+}\right)-$ $u\left(t_{k}^{-}\right), \Delta \phi_{p}\left(u^{\Delta}\left(t_{k}\right)\right)=\phi_{p}\left(u^{\Delta}\left(t_{k}^{+}\right)\right)-\phi_{p}\left(u^{\Delta}\left(t_{k}^{-}\right)\right), u\left(t_{k}^{+}\right)$and $u\left(t_{k}^{-}\right)$represent the right-hand limit and left-hand limit of the function $u(t)$ at $t=t_{k}, k=1,2, \ldots, n$.

In this paper we assume that

(A1) $f \in \mathcal{C}\left([0,1]_{\mathbb{T}} \times \mathbb{R}^{+} \times \mathbb{R}, \mathbb{R}^{+}\right)$,

(A2) $\alpha_{j} \in[0, \infty), \beta_{j} \in[0, \infty), j=1,2, \ldots, m-2$, with $0<\sum_{j=1}^{m-2} \alpha_{j}<1$ and $0<\sum_{j=1}^{m-2} \beta_{j}<1$,

(A3) $q \in \mathcal{C}\left([0,1]_{\mathbb{T}}\right)$ is nonnegative and there exists an integer $l \geq 3, \frac{1}{l}, 1-\frac{1}{l} \in \mathbb{T}$ such that $\int_{\frac{1}{l}}^{1-\frac{1}{7}} q(t) \nabla t>0$

(A4) $I_{k} \in \mathcal{C}\left(\mathbb{R}^{+}, \mathbb{R}^{+}\right)$is a bounded function, $\bar{I}_{k} \in \mathcal{C}\left(\mathbb{R}^{+} \times \mathbb{R}, \mathbb{R}^{+}\right), k=1,2, \ldots, n$,

(A5) $\max \left\{G_{1}, G_{2}, \ldots, G_{n}\right\} \leq \frac{l b_{1}}{n}$, where $G_{k}=\sup _{u \in[0, \infty)} I_{k}(u)$ and $b_{1}>0$ is a constant which is given by Theorem 3.1.

In this study, by employing Bai-Ge's fixed point theorem [16], we get the existence of at least three positive solutions for boundary value problem (1.1). In fact, our result is also new when $\mathbb{T}=\mathbb{R}$ (the differential case) and $\mathbb{Z}$ (the discrete case). Therefore, the result can be considered as a contribution to this field.

This paper is organized as follows. In Section 2, we give some definitions and preliminary lemmas which are key tools for our proof. The main results are given in Section 3. Finally, in Section 4, we give an example to demonstrate our results.

\section{Preliminaries}

In this section, we give some lemmas which are useful for our main results.

Throughout the rest of this paper, we always assume that the points of impulse $t_{k}$ are right dense for each $k=1,2, \ldots, n$. Let $J=[0,1]_{\mathbb{T}}, J_{0}=\left[0, t_{1}\right]_{\mathbb{T}}, J_{1}=\left(t_{1}, t_{2}\right]_{\mathbb{T}}, \ldots, J_{n-1}=$ $\left(t_{n-1}, t_{n}\right]_{\mathbb{T}}, J_{n}=\left(t_{n}, 1\right]_{\mathbb{T}}, J^{\prime}=J \backslash\left\{t_{1}, t_{2}, \ldots, t_{n}\right\}$.

Set

$$
\begin{aligned}
P C(J)= & \left\{u:[0,1]_{\mathbb{T}} \longrightarrow \mathbb{R} ; u \in C\left(J^{\prime}\right), u\left(t_{k}^{+}\right) \text {and } u\left(t_{k}^{-}\right)\right. \text {exist; } \\
& \text { and } \left.u\left(t_{k}^{-}\right)=u\left(t_{k}\right), 1 \leq k \leq n\right\}, \\
P C^{1}(J)= & \left\{u \in P C(J): u^{\Delta} \in C\left(J^{\prime}\right), u^{\Delta}\left(t_{k}^{+}\right) \text {and } u^{\Delta}\left(t_{k}^{-}\right)\right. \text {exist; } \\
& \text { and } \left.u^{\Delta}\left(t_{k}^{-}\right)=u^{\Delta}\left(t_{k}\right), 1 \leq k \leq n\right\} .
\end{aligned}
$$


Obviously, $P C(J)$ and $P C^{1}(J)$ are Banach spaces with the norms

$$
\|u\|_{P C}=\max _{t \in[0,1]_{T}}|u(t)|, \quad\|u\|_{P C^{1}}=\max \left\{\|u\|_{P C},\left\|u^{\Delta}\right\|_{P C}\right\},
$$

respectively. A function $u \in P C^{1}(J) \cap C^{2}\left(J^{\prime}\right)$ is called a solution to (1.1) if it satisfies all the equations of (1.1).

Define the cone $P \subset P C^{1}(J)$ by

$$
\begin{aligned}
P= & \left\{u \in P C^{1}(J): u(t) \geq 0, u \text { is concave on } J_{k}(0 \leq k \leq n)\right. \\
& \text { and } \left.u(t), u^{\Delta}(t) \text { are non-increasing on }[0,1]_{\mathbb{T}}\right\} .
\end{aligned}
$$

Lemma 2.1 If $u$ is continuous, nonnegative and concave on $[0,1]_{\mathbb{T}}$, then

$$
u\left(1-\frac{1}{l}\right) \geq \frac{1}{l} \max _{t \in[0,1]_{\mathbb{T}}}|u(t)|
$$

where $l \geq 3$.

Proof Suppose that $\max _{t \in[0,1]_{\mathbb{T}}}|u(t)|=u(\sigma)$, where $\sigma \in[0,1]_{\mathbb{T}}$. Since $u$ is concave and nonnegative,

$$
\begin{aligned}
u\left(1-\frac{1}{l}\right) & =u\left(\frac{1+\sigma}{l(1+\sigma)}(l-1)(1-\sigma)+\frac{l-1}{l} \sigma\right) \\
& \geq \frac{1+\sigma}{l(1+\sigma)} u((l-1)(1-\sigma))+\left(\frac{l-1}{l}\right) u(\sigma) \\
& \geq\left(\frac{l-1}{l}\right) u(\sigma) \\
& \geq \frac{1}{l} u(\sigma) \\
& \geq \frac{1}{l} \max _{t \in[0,1]_{\mathbb{T}}}|u(t)| .
\end{aligned}
$$

Lemma 2.2 If $u \in P, l \geq 3$, then

$$
\min _{t \in\left[\frac{1}{l}, 1-\frac{1}{l}\right]_{\mathbb{T}}}|u(t)| \geq \frac{1}{l} \max _{t \in[0,1]_{\mathbb{T}}}|u(t)|-\frac{1}{l} \sum_{i=1}^{n}\left|\Delta u\left(t_{i}\right)\right| .
$$

Proof Let

$$
v(t)= \begin{cases}u(t)-\sum_{k=1}^{n}\left|\Delta u\left(t_{k}\right)\right|, & t \in J_{0}, \\ u(t)-\sum_{k=2}^{n}\left|\Delta u\left(t_{k}\right)\right|, & t \in J_{1}, \\ \vdots & \\ u(t)-\left|\Delta u\left(t_{n}\right)\right|, & t \in J_{n-1}, \\ u(t), & t \in J_{n} .\end{cases}
$$


Note that $u^{\Delta}(t)$ is non-increasing on $[0,1]_{\mathbb{T}}$, hence $v \in \mathcal{C}[0,1]_{\mathbb{T}}$ and $v$ is concave on $[0,1]_{\mathbb{T}}$. By Lemma 2.1, we have

$$
\min _{t \in\left[\frac{1}{l}, 1-\frac{1}{l}\right]_{\mathbb{T}}}|v(t)|=v\left(1-\frac{1}{l}\right) \geq \frac{1}{l} \max _{t \in[0,1]_{\mathbb{T}}}|v(t)| .
$$

Moreover, $u(t)$ is non-increasing on $[0,1]_{\mathbb{T}}$, and we have

$$
\begin{aligned}
\max _{t \in[0,1]_{\mathbb{T}}}|v(t)| & =v(0) \\
= & u(0)-\sum_{k=1}^{n}\left|\Delta u\left(t_{k}\right)\right| \\
& =\max _{t \in[0,1]_{\mathbb{T}}}|u(t)|-\sum_{k=1}^{n}\left|\Delta u\left(t_{k}\right)\right|, \\
\min _{t \in\left[\frac{1}{7}, 1-\frac{1}{l}\right]_{\mathbb{T}}}|v(t)| & =v\left(1-\frac{1}{l}\right) \\
& =u\left(1-\frac{1}{l}\right)-\sum_{1-\frac{1}{l}<t_{k}<1}\left|\Delta u\left(t_{k}\right)\right| \\
& =\min _{t \in\left[\frac{1}{l}, 1-\frac{1}{l}\right]_{\mathbb{T}}}|u(t)|-\sum_{1-\frac{1}{l}<t_{k}<1}\left|\Delta u\left(t_{k}\right)\right| .
\end{aligned}
$$

Hence,

$$
\begin{aligned}
\min _{t \in\left[\frac{1}{l}, 1-\frac{1}{l}\right]_{\mathbb{T}}}|u(t)| & =\min _{t \in\left[\frac{1}{l}, 1-\frac{1}{l}\right]_{\mathbb{T}}}|v(t)|+\sum_{0<t_{k}<\frac{1}{l}}\left|\Delta u\left(t_{k}\right)\right| \\
& \geq \frac{1}{l} \max _{t \in[0,1]_{\mathbb{T}}}|v(t)| \\
& =\frac{1}{l} \max _{t \in[0,1]_{\mathbb{T}}}|u(t)|-\frac{1}{l} \sum_{k=1}^{n}\left|\Delta u\left(t_{k}\right)\right| .
\end{aligned}
$$

Lemma 2.3 Assume that (A1)-(A4) hold. Then $u \in P C^{1}(J) \cap C^{2}\left(J^{\prime}\right)$ is a solution to problem (1.1) if and only if $u \in P C^{1}(J)$ is a solution to the integral equation:

$$
\begin{aligned}
u(t)= & \int_{t}^{1} \phi_{q}\left(\int_{0}^{s} q(\tau) f\left(\tau, u(\tau), u^{\Delta}(\tau)\right) \nabla \tau+\sum_{0<t_{k}<s} \bar{I}_{k}\left(u\left(t_{k}\right), u^{\Delta}\left(t_{k}\right)\right)+A\right) \Delta s \\
& +\frac{\sum_{j=1}^{m-2} \beta_{j}}{1-\sum_{i=1}^{m-2} \beta_{i}} \int_{\eta_{j}}^{1} \phi_{q}\left(\int_{0}^{s} q(\tau) f\left(\tau, u(\tau), u^{\Delta}(\tau)\right) \nabla \tau\right. \\
& \left.+\sum_{0<t_{k}<s} \bar{I}_{k}\left(u\left(t_{k}\right), u^{\Delta}\left(t_{k}\right)\right)+A\right) \Delta s \\
& +\frac{1}{1-\sum_{i=1}^{m-2} \beta_{i}} \sum_{j=1}^{m-2} \beta_{j} \sum_{\eta_{j}<t_{k}<1} I_{k}\left(u\left(t_{k}\right)\right)+\sum_{t<t_{k}<1} I_{k}\left(u\left(t_{k}\right)\right),
\end{aligned}
$$


where

$$
A=\frac{1}{1-\sum_{i=1}^{m-2} \alpha_{i}} \sum_{j=1}^{m-2} \alpha_{j}\left[\int_{0}^{\xi_{j}} q(s) f\left(s, u(s), u^{\Delta}(s)\right) \nabla s+\sum_{0<t_{k}<\xi_{j}} \bar{I}_{k}\left(u\left(t_{k}\right), u^{\Delta}\left(t_{k}\right)\right)\right] .
$$

Proof First, suppose that $u \in P C^{1}(J) \cap C^{2}\left(J^{\prime}\right)$ is a solution to problem (1.1). Then

$$
\left(\phi_{p}\left(u^{\Delta}(t)\right)\right)^{\nabla}+q(t) f\left(t, u(t), u^{\Delta}(t)\right)=0, \quad t \neq t_{k}, k=1,2, \ldots, n .
$$

So,

$$
\begin{aligned}
& \phi_{p}\left(u^{\Delta}\left(t_{1}^{-}\right)\right)-\phi_{p}\left(u^{\Delta}(0)\right)=-\int_{0}^{t_{1}} q(s) f\left(s, u(s), u^{\Delta}(s)\right) \nabla s, \\
& \phi_{p}\left(u^{\Delta}(t)\right)-\phi_{p}\left(u^{\Delta}\left(t_{1}^{+}\right)\right)=-\int_{t_{1}}^{t} q(s) f\left(s, u(s), u^{\Delta}(s)\right) \nabla s, \quad t \in J_{1} .
\end{aligned}
$$

Thus,

$$
\phi_{p}\left(u^{\Delta}(t)\right)=\phi_{p}\left(u^{\Delta}(0)\right)-\int_{0}^{t} q(s) f\left(s, u(s), u^{\Delta}(s)\right) \nabla s-\bar{I}_{1}\left(u\left(t_{1}\right), u^{\Delta}\left(t_{1}\right)\right), \quad t \in J_{1} .
$$

Repeating the above process, for $t \in[0,1]_{\mathbb{T}}$, we have

$$
\phi_{p}\left(u^{\Delta}(t)\right)=\phi_{p}\left(u^{\Delta}(0)\right)-\int_{0}^{t} q(s) f\left(s, u(s), u^{\Delta}(s)\right) \nabla s-\sum_{0<t_{k}<t} \bar{I}_{k}\left(u\left(t_{k}\right), u^{\Delta}\left(t_{k}\right)\right),
$$

and taking $t=\xi_{j}$ in (2.2), we have

$$
\phi_{p}\left(u^{\Delta}\left(\xi_{j}\right)\right)=\phi_{p}\left(u^{\Delta}(0)\right)-\int_{0}^{\xi_{j}} q(s) f\left(s, u(s), u^{\Delta}(s)\right) \nabla s-\sum_{0<t_{k}<\xi_{j}} \bar{I}_{k}\left(u\left(t_{k}\right), u^{\Delta}\left(t_{k}\right)\right) .
$$

So, we get

$$
\begin{aligned}
\sum_{j=1}^{m-2} \alpha_{j} \phi_{p}\left(u^{\Delta}\left(\xi_{j}\right)\right)= & \sum_{j=1}^{m-2} \alpha_{j} \phi_{p}\left(u^{\Delta}(0)\right)-\sum_{j=1}^{m-2} \alpha_{j} \int_{0}^{\xi_{j}} q(s) f\left(s, u(s), u^{\Delta}(s)\right) \nabla s \\
& -\sum_{j=1}^{m-2} \alpha_{j} \sum_{0<t_{k}<\xi_{j}} \bar{I}_{k}\left(u\left(t_{k}\right), u^{\Delta}\left(t_{k}\right)\right) .
\end{aligned}
$$

Since $\phi_{p}\left(u^{\Delta}(0)\right)=\sum_{j=1}^{m-2} \alpha_{j} \phi_{p}\left(u^{\Delta}\left(\xi_{j}\right)\right)$, we have

$$
\begin{aligned}
\phi_{p}\left(u^{\Delta}(0)\right) & =-\frac{\sum_{j=1}^{m-2} \alpha_{j}}{1-\sum_{i=1}^{m-2} \alpha_{i}}\left[\int_{0}^{\xi_{j}} q(s) f\left(s, u(s), u^{\Delta}(s)\right) \nabla s+\sum_{0<t_{k}<\xi_{j}} \bar{I}_{k}\left(u\left(t_{k}\right), u^{\Delta}\left(t_{k}\right)\right)\right] \\
& =-A .
\end{aligned}
$$


Substituting (2.3) into (2.2), we get

$$
\phi_{p}\left(u^{\Delta}(t)\right)=-\left[\int_{0}^{t} q(s) f\left(s, u(s), u^{\Delta}(s)\right) \nabla s+\sum_{0<t_{k}<t} \bar{I}_{k}\left(u\left(t_{k}\right), u^{\Delta}\left(t_{k}\right)\right)+A\right]
$$

which implies that

$$
u^{\Delta}(t)=-\phi_{q}\left(\int_{0}^{t} q(s) f\left(s, u(s), u^{\Delta}(s)\right) \nabla s+\sum_{0<t_{k}<t} \bar{I}_{k}\left(u\left(t_{k}\right), u^{\Delta}\left(t_{k}\right)\right)+A\right) .
$$

On the other hand, note that

$$
\begin{aligned}
& u\left(t_{n}^{-}\right)-u(t)=\int_{t}^{t_{n}} u^{\Delta}(s) \Delta s, \\
& u(1)-u\left(t_{n}^{+}\right)=\int_{t_{n}}^{1} u^{\Delta}(s) \Delta s, \quad t \in J_{n-1} .
\end{aligned}
$$

So that we have

$$
u(t)=u(1)-\int_{t}^{1} u^{\Delta}(s) \Delta s+I_{n}\left(u\left(t_{n}\right)\right), \quad t \in J_{n-1} .
$$

Repeating the above process again for $t \in[0,1]_{\mathbb{T}}$, we obtain

$$
u(t)=u(1)-\int_{t}^{1} u^{\Delta}(s) \Delta s+\sum_{t<t_{k}<1} I_{k}\left(u\left(t_{k}\right)\right)
$$

Substituting (2.4) into (2.5), we get

$$
\begin{aligned}
u(t)= & u(1)+\sum_{t<t_{k}<1} I_{k}\left(u\left(t_{k}\right)\right) \\
& +\int_{t}^{1} \phi_{q}\left(\int_{0}^{s} q(\tau) f\left(\tau, u(\tau), u^{\Delta}(\tau)\right) \nabla \tau+\sum_{0<t_{k}<s} \bar{I}_{k}\left(u\left(t_{k}\right), u^{\Delta}\left(t_{k}\right)\right)+A\right) \Delta s,
\end{aligned}
$$

and taking $t=\eta_{j}$ in (2.6), we get

$$
\begin{aligned}
u\left(\eta_{j}\right)= & u(1)+\sum_{\eta_{j}<t_{k}<1} I_{k}\left(u\left(t_{k}\right)\right) \\
& +\int_{\eta_{j}}^{1} \phi_{q}\left(\int_{0}^{s} q(\tau) f\left(\tau, u(\tau), u^{\Delta}(\tau)\right) \nabla \tau+\sum_{0<t_{k}<s} \bar{I}_{k}\left(u\left(t_{k}\right), u^{\Delta}\left(t_{k}\right)\right)+A\right) \Delta s .
\end{aligned}
$$


So,

$$
\begin{aligned}
& \sum_{j=1}^{m-2} \beta_{j} u\left(\eta_{j}\right) \\
& =u(1) \sum_{j=1}^{m-2} \beta_{j}+\sum_{j=1}^{m-2} \beta_{j} \sum_{\eta_{j}<t_{k}<1} I_{k}\left(u\left(t_{k}\right)\right) \\
& \quad+\sum_{j=1}^{m-2} \beta_{j} \int_{\eta_{j}}^{1} \phi_{q}\left(\int_{0}^{s} q(\tau) f\left(\tau, u(\tau), u^{\Delta}(\tau)\right) \nabla \tau+\sum_{0<t_{k}<s} \bar{I}_{k}\left(u\left(t_{k}\right), u^{\Delta}\left(t_{k}\right)\right)+A\right) \Delta s
\end{aligned}
$$

Since

$$
\begin{aligned}
u(1)= & \sum_{j=1}^{m-2} \beta_{j} u\left(\eta_{j}\right), \\
= & \frac{\sum_{j=1}^{m-2} \beta_{j}}{1-\sum_{i=1}^{m-2} \beta_{i}} \int_{\eta_{j}}^{1} \phi_{q}\left(\int_{0}^{s} q(\tau) f\left(\tau, u(\tau), u^{\Delta}(\tau)\right) \nabla \tau\right. \\
& \left.+\sum_{0<t_{k}<s} \bar{I}_{k}\left(u\left(t_{k}\right), u^{\Delta}\left(t_{k}\right)\right)+A\right) \Delta s+\frac{1}{1-\sum_{i=1}^{m-2} \beta_{i}} \sum_{j=1}^{m-2} \beta_{j} \sum_{\eta_{j}<t_{k}<1} I_{k}\left(u\left(t_{k}\right)\right) .
\end{aligned}
$$

Substituting (2.7) into (2.6), we get (2.1), which completes the proof of sufficiency.

Conversely, if $u(t) \in P C^{1}(J)$ is a solution to (2.1), apparently

$$
\Delta u\left(t_{k}\right)=-I_{k}\left(u\left(t_{k}\right)\right), \quad k=1,2, \ldots, n .
$$

The $\Delta$-derivative of (2.1) implies that for $t \neq t_{k}$,

$$
\begin{aligned}
& u^{\Delta}(t)=-\phi_{q}\left(\int_{0}^{t} q(s) f\left(s, u(s), u^{\Delta}(s)\right) \nabla s+\sum_{0<t_{k}<t} \bar{I}_{k}\left(u\left(t_{k}\right), u^{\Delta}\left(t_{k}\right)\right)+A\right), \\
& \left(\phi_{p}\left(u^{\Delta}(t)\right)\right)^{\nabla}=-q(t) f\left(t, u(t), u^{\Delta}(t)\right) .
\end{aligned}
$$

Hence $u \in C^{2}\left(J^{\prime}\right)$, and

$$
\begin{array}{ll}
\Delta \phi_{p}\left(u^{\Delta}\left(t_{k}\right)\right)=-\bar{I}_{k}\left(u\left(t_{k}\right), u^{\Delta}\left(t_{k}\right)\right), & k=1,2, \ldots, n, \\
\phi_{p}\left(u^{\Delta}(0)\right)=\sum_{j=1}^{m-2} \alpha_{j} \phi_{p}\left(u^{\Delta}\left(\xi_{j}\right)\right), & u(1)=\sum_{j=1}^{m-2} \beta_{j} u\left(\eta_{j}\right) .
\end{array}
$$

The proof is complete.

Now define an operator $T: P \longrightarrow P C^{1}(J)$ by

$$
\begin{aligned}
T u(t)= & \int_{t}^{1} \phi_{q}\left(\int_{0}^{s} q(\tau) f\left(\tau, u(\tau), u^{\Delta}(\tau)\right) \nabla \tau+\sum_{0<t_{k}<s} \bar{I}_{k}\left(u\left(t_{k}\right), u^{\Delta}\left(t_{k}\right)\right)+A\right) \Delta s \\
& +\frac{\sum_{j=1}^{m-2} \beta_{j}}{1-\sum_{i=1}^{m-2} \beta_{i}} \int_{\eta_{j}}^{1} \phi_{q}\left(\int_{0}^{s} q(\tau) f\left(\tau, u(\tau), u^{\Delta}(\tau)\right) \nabla \tau\right.
\end{aligned}
$$




$$
\begin{aligned}
& \left.+\sum_{0<t_{k}<s} \bar{I}_{k}\left(u\left(t_{k}\right), u^{\Delta}\left(t_{k}\right)\right)+A\right) \Delta s \\
& +\frac{1}{1-\sum_{i=1}^{m-2} \beta_{i}} \sum_{j=1}^{m-2} \beta_{j} \sum_{\eta_{j}<t_{k}<1} I_{k}\left(u\left(t_{k}\right)\right)+\sum_{t<t_{k}<1} I_{k}\left(u\left(t_{k}\right)\right) .
\end{aligned}
$$

Lemma 2.4 Assume that (A1)-(A4) hold. Then $T: P \rightarrow P$ is a completely continuous operator.

Proof From the definition of $T$, it is clear that $T(P) \subset P$. On the other hand, by conditions (A1)-(A4) and the definition of $T u(t)$, it is clear that $T: P \rightarrow P$ is continuous.

Let $\Omega \subset P$ be bounded, i.e., there exists a positive constant $R$ such that

$$
\Omega \subset\left\{u \in P:\|u\|_{P C^{1}} \leq R\right\} .
$$

Let

$$
\begin{aligned}
& B_{1}=\max _{(t, u, v) \in[0,1]_{\mathbb{T}} \times[0, R] \times[0, R]} f(t, u, v), \\
& B_{2}=\max _{1 \leq k \leq n}\left\{\max _{u \in[0, R]} I_{k}(u)\right\}, \\
& B_{3}=\max _{1 \leq k \leq n}\left\{\max _{(u, v) \in[0, R] \times[0, R]} \bar{I}_{k}(u, v)\right\}, \\
& R_{1}=\max _{t \in[0,1]_{\mathbb{T}}} q(t) .
\end{aligned}
$$

For all $u \in \Omega$, we have

$$
A \leq \frac{1}{1-\sum_{j=1}^{m-2} \alpha_{j}} \sum_{j=1}^{m-2} \alpha_{j}\left[B_{1} R_{1}+n B_{3}\right] .
$$

Hence,

$$
\begin{aligned}
& |T u(t)| \leq \frac{1}{1-\sum_{j=1}^{m-2} \beta_{j}}\left[\phi_{q}\left(\frac{B_{1} R_{1}+n B_{3}}{1-\sum_{j=1}^{m-2} \alpha_{j}}\right)+n B_{2}\right], \\
& \left|(T u)^{\Delta}(t)\right| \leq \phi_{q}\left[\frac{B_{1} R_{1}+n B_{3}}{1-\sum_{j=1}^{m-2} \alpha_{j}}\right], \\
& \left|\left(\phi_{p}(T u)^{\Delta}(t)\right)^{\nabla}\right| \leq R_{1} B_{1}, \quad t \neq t_{k}, k=1,2, \ldots, n .
\end{aligned}
$$

So, $T u$ and $(T u)^{\Delta}$ are bounded on $J$ and equicontinuous on each $J_{k}(k=0,1, \ldots, n)$. This implies that $T \Omega$ is relatively compact. Therefore, the operator $T: P \rightarrow P$ is completely continuous.

\section{Main results}

In this section we state and prove our main result. Define the following convex sets:

$$
\begin{aligned}
& P(\varphi, r ; \omega, L)=\{u \in P: \varphi(u)<r, \omega(u)<L\}, \\
& \bar{P}(\varphi, r ; \omega, L)=\{u \in P: \varphi(u) \leq r, \omega(u) \leq L\},
\end{aligned}
$$




$$
\begin{aligned}
& P(\varphi, r ; \omega, L ; \psi, a)=\{u \in P: \varphi(u)<r, \omega(u)<L, \psi(u)>a\}, \\
& \bar{P}(\varphi, r ; \omega, L ; \psi, a)=\{u \in P: \varphi(u) \leq r, \omega(u) \leq L, \psi(u) \geq a\} .
\end{aligned}
$$

The following assumptions as regards the nonnegative continuous convex functions $\varphi$, $\omega$ are used:

(B1) there exists $M>0$ such that $\|x\| \leq M \max \{\varphi(x), \omega(x)\}$ for all $x \in P$;

(B2) $P(\varphi, r ; \omega, L) \neq \emptyset$ for any $r>0$ and $L>0$.

To prove our main result, we need the following fixed point theorem due to Bai and Ge in [16].

Lemma 3.1 [16] Let $P$ be a cone in a real Banach space $\mathbb{E}$, and let $r_{2} \geq d>b>r_{1}>0$, $L_{2} \geq L_{1}>0$. Assume that $\varphi$ and $\omega$ are nonnegative continuous convex functions satisfying (B1) and (B2), $\psi$ is a nonnegative continuous concave function on $P$ such that $\psi(u) \leq \varphi(u)$ for all $u \in \bar{P}\left(\varphi, r_{2} ; \omega, L_{2}\right)$ and $T: \bar{P}\left(\varphi, r_{2} ; \omega, L_{2}\right) \rightarrow \bar{P}\left(\varphi, r_{2} ; \omega, L_{2}\right)$ is a completely continuous operator. Suppose that

(B3) $\left\{u \in \bar{P}\left(\varphi, d ; \omega, L_{2} ; \psi, b\right): \psi(u)>b\right\} \neq \emptyset, \psi(T u)>b$ for $u \in \bar{P}\left(\varphi, d ; \omega, L_{2} ; \psi, b\right)$,

(B4) $\varphi(T u)<r_{1}, \omega(T u)<L_{1}$ for all $u \in \bar{P}\left(\varphi, r_{1} ; \omega, L_{1}\right)$,

(B5) $\psi(T u)>b$ for all $u \in \bar{P}\left(\varphi, r_{2} ; \omega, L_{2} ; \psi, b\right)$ with $\varphi(T u)>d$.

Then $T$ has at least three fixed points $u_{1}, u_{2}$ and $u_{3} \in \bar{P}\left(\varphi, r_{2} ; \omega, L_{2}\right)$ with

$$
\begin{aligned}
& u_{1} \in P\left(\varphi, r_{1} ; \omega, L_{1}\right), \quad u_{2} \in\left\{\bar{P}\left(\varphi, r_{2} ; \omega, L_{2} ; \psi, b\right): \psi(u)>b\right\} \\
& u_{3} \in \bar{P}\left(\varphi, r_{2} ; \omega, L_{2}\right) \backslash\left(\bar{P}\left(\varphi, r_{2} ; \omega, L_{2} ; \psi, b\right) \cup \bar{P}\left(\varphi, r_{1} ; \omega, L_{1}\right)\right) .
\end{aligned}
$$

Define nonnegative continuous functionals $\varphi, \omega$ and $\psi$ by

$$
\varphi(u)=\max _{t \in[0,1]_{\mathbb{T}}}|u(t)|, \quad \omega(u)=\max _{t \in[0,1]_{\mathbb{T}}}\left|u^{\Delta}(t)\right|, \quad \psi(u)=\min _{t \in\left[\frac{1}{l}, 1-\frac{1}{l}\right]_{\mathbb{T}}}|u(t)| \quad \text { for } u \in P .
$$

Then, on the cone $P, \psi$ is a concave functional, $\varphi$ and $\omega$ are convex functionals satisfying (B1) and (B2).

Let

$$
\begin{aligned}
& H=\frac{1}{1-\sum_{j=1}^{m-2} \beta_{j}}\left[\phi_{q}\left(\frac{R_{1}+n}{1-\sum_{j=1}^{m-2} \alpha_{j}}\right)+n\right], \\
& L=\phi_{q}\left(\frac{R_{1}+n}{1-\sum_{j=1}^{m-2} \alpha_{j}}\right), \\
& N=\phi_{q}\left(\int_{1 / l}^{1-1 / l} q(r) \nabla r\right) .
\end{aligned}
$$

Theorem 3.1 Assume that (A1)-(A4) hold. There exist constants $r_{2} \geq 2 l b_{1}>b_{1}>r_{1}>0$, $L_{2} \geq L_{1}>0$ and the following assumptions are satisfied:

(A5) $\max \left\{f(t, u, v), \bar{I}_{k}(u, v)\right\}<\min \left\{\phi_{p}\left(\frac{r_{1}}{H}\right), \phi_{p}\left(\frac{L_{1}}{L}\right)\right\}, I_{k}(u) \leq \frac{r_{1}}{H}$ for

$$
(t, u, v) \in[0,1]_{\mathbb{T}} \times\left[0, r_{1}\right] \times\left[0, L_{1}\right], 1 \leq k \leq n ;
$$

(A6) $f(t, u, v)>\phi_{p}\left(\frac{l b_{1}}{N}\right)$ for $(t, u, v) \in\left[\frac{1}{l}, 1-\frac{1}{l}\right]_{\mathbb{T}} \times\left[b_{1}, 2 l b_{1}\right] \times\left[0, L_{2}\right]$;

(A7) $\max \left\{f(t, u, v), \bar{I}_{k}(u, v)\right\}<\min \left\{\phi_{p}\left(\frac{r_{2}}{H}\right), \phi_{p}\left(\frac{L_{2}}{L}\right)\right\}, I_{k}(u) \leq \frac{r_{2}}{H}$ for $(t, u, v) \in[0,1]_{\mathbb{T}} \times\left[0, r_{2}\right] \times\left[0, L_{2}\right], 1 \leq k \leq n$. 
Then problem (1.1) possesses at least three positive solutions $u_{1}, u_{2}$ and $u_{3}$ such that

$$
\begin{aligned}
& \max _{t \in[0,1]_{\mathbb{T}}} u_{1}(t)<r_{1}, \quad \max _{t \in[0,1]_{\mathbb{T}}}\left|u_{1}^{\Delta}(t)\right|<L_{1} ; \\
& b_{1}<\min _{t \in\left[\frac{1}{l}, 1-\frac{1}{l}\right]_{\mathbb{T}}} u_{2}(t) \leq \max _{t \in[0,1]_{\mathbb{T}}} u_{2}(t) \leq r_{2}, \quad \max _{t \in[0,1]_{\mathbb{T}}}\left|u_{2}^{\Delta}(t)\right| \leq L_{2} ; \\
& r_{1}<\max _{t \in[0,1]_{\mathbb{T}}} u_{3}(t)<r_{2}, \quad \min _{t \in\left[\frac{1}{l}, 1-\frac{1}{l}\right]_{\mathbb{T}}} u_{3}(t)<b_{1}, \quad \max _{t \in[0,1]_{\mathbb{T}}}\left|u_{3}^{\Delta}(t)\right| \leq L_{2} .
\end{aligned}
$$

Proof Problem (1.1) has a solution $u=u(t)$ if and only if $u$ solves the operator equation $u=T u$. We have shown $T: P \rightarrow P$ is completely continuous by Lemma 2.4 . We now verify that all the conditions of Lemma 3.1 are satisfied. The proof is divided into four steps.

Step 1. First we show that

$$
T: \bar{P}\left(\varphi, r_{2} ; \omega, L_{2}\right) \rightarrow \bar{P}\left(\varphi, r_{2} ; \omega, L_{2}\right) .
$$

If $u \in \bar{P}\left(\varphi, r_{2} ; \omega, L_{2}\right)$, then $\varphi(u) \leq r_{2}, \omega(u) \leq L_{2}$, and by assumption (A7), we have

$$
\begin{aligned}
A & \leq \frac{\sum_{j=1}^{m-2} \alpha_{j}}{1-\sum_{i=1}^{m-2} \alpha_{i}}\left[\int_{0}^{1} q(s) f\left(s, u(s), u^{\Delta}(s)\right) \nabla s+\sum_{k=1}^{n} \bar{I}_{k}\left(u\left(t_{k}\right), u^{\Delta}\left(t_{k}\right)\right)\right] \\
& \leq \frac{\sum_{j=1}^{m-2} \alpha_{j}}{1-\sum_{i=1}^{m-2} \alpha_{i}} \min \left\{\phi_{p}\left(\frac{r_{2}}{H}\right), \phi_{p}\left(\frac{L_{2}}{L}\right)\right\}\left(R_{1}+n\right) .
\end{aligned}
$$

Hence,

$$
\begin{aligned}
\varphi(T u)= & \max _{t \in[0,1]_{\mathbb{T}}}|u(t)|=(T u)(0) \\
= & \int_{0}^{1} \phi_{q}\left(\int_{0}^{s} q(\tau) f\left(\tau, u(\tau), u^{\Delta}(\tau)\right) \nabla \tau+\sum_{0<t_{k}<s} \bar{I}_{k}\left(u\left(t_{k}\right), u^{\Delta}\left(t_{k}\right)\right)+A\right) \Delta s \\
& +\sum_{k=1}^{n} I_{k}\left(u\left(t_{k}\right)\right) \\
& +\frac{\sum_{j=1}^{m-2} \beta_{j}}{1-\sum_{i=1}^{m-2} \beta_{i}} \int_{\eta_{j}}^{1} \phi_{q}\left(\int_{0}^{s} q(\tau) f\left(\tau, u(\tau), u^{\Delta}(\tau)\right) \nabla \tau\right. \\
& \left.+\sum_{0<t_{k}<s} \bar{I}_{k}\left(u\left(t_{k}\right), u^{\Delta}\left(t_{k}\right)\right)+A\right) \Delta s \\
& +\frac{1}{1-\sum_{i=1}^{m-2} \beta_{i}} \sum_{j=1}^{m-2} \beta_{j} \sum_{\eta_{j}<t_{k}<1} I_{k}\left(u\left(t_{k}\right)\right) \\
\leq & \frac{1}{1-\sum_{j=1}^{m-2} \beta_{j}} \int_{0}^{1} \phi_{q}\left(\int_{0}^{1} q(\tau) f\left(\tau, u(\tau), u^{\Delta}(\tau)\right) \nabla \tau\right. \\
& \left.+\sum_{k=1}^{n} \bar{I}_{k}\left(u\left(t_{k}\right), u^{\Delta}\left(t_{k}\right)\right)+A\right) \Delta s \\
& +\frac{1}{1-\sum_{j=1}^{m-2} \beta_{j}} \sum_{k=1}^{n} I_{k}\left(u\left(t_{k}\right)\right)
\end{aligned}
$$




$$
\begin{aligned}
& \leq \frac{r_{2}}{H} \frac{1}{1-\sum_{j=1}^{m-2} \beta_{j}}\left[\phi_{q}\left(\frac{R_{1}+n}{1-\sum_{j=1}^{m-2} \alpha_{j}}\right)+n\right] \\
& =r_{2}, \\
\omega(T u) & =\max _{t \in[0,1]_{\mathbb{T}}}\left|(T u)^{\Delta}(t)\right|=\left|(T u)^{\Delta}(1)\right| \\
& \leq \frac{L_{2}}{L} \phi_{q}\left(\frac{R_{1}+n}{1-\sum_{j=1}^{m-2} \alpha_{j}}\right) \\
& =L_{2} .
\end{aligned}
$$

So, (3.1) holds.

Step 2. We show that condition (B3) in Lemma 3.1 holds. We choose $u(t)=\frac{2 l+1}{2} b_{1}$ for $t \in[0,1]_{\mathbb{T}}$. It is easy to see that $u(t) \in \bar{P}\left(\varphi, 2 l b_{1} ; \omega, L_{2} ; \psi, b_{1}\right), \psi(u)=u\left(1-\frac{1}{l}\right)>b_{1}$ and consequently $\left\{u \in \bar{P}\left(\varphi, 2 l b_{1} ; \omega, L_{2} ; \psi, b_{1}\right): \psi(u)>b_{1}\right\} \neq \emptyset$. Thus, for $u \in \bar{P}\left(\varphi, 2 l b_{1} ; \omega, L_{2} ; \psi, b_{1}\right)$, there is $b_{1} \leq u(t) \leq 2 l b_{1}$ for $t \in\left[\frac{1}{l}, 1-\frac{1}{l}\right]_{\mathbb{T}}$. By condition (A6), we have

$$
\begin{aligned}
\psi(T u) & =\min _{t \in\left[\frac{1}{l}, 1-\frac{1}{l}\right]_{\mathbb{T}}}|T u(t)|=(T u)\left(1-\frac{1}{l}\right) \\
& \geq \int_{1-1 / l}^{1} \phi_{q}\left(\int_{0}^{s} q(\tau) f\left(\tau, u(\tau), u^{\Delta}(\tau)\right) \nabla \tau\right) \Delta s \\
& \geq \int_{1-1 / l}^{1} \phi_{q}\left(\int_{0}^{1-1 / l} q(\tau) f\left(\tau, u(\tau), u^{\Delta}(\tau)\right) \nabla \tau\right) \Delta s \\
& \geq \frac{1}{l} \phi_{q}\left(\int_{1 / l}^{1-1 / l} q(\tau) f\left(\tau, u(\tau), u^{\Delta}(\tau)\right) \nabla \tau\right) \\
& \geq \frac{b_{1}}{N} \phi_{q}\left(\int_{1 / l}^{1-1 / l} q(r) \nabla r\right) \\
& =b_{1} .
\end{aligned}
$$

Therefore,

$$
\psi(T u)>b_{1}, \quad \forall u \in \bar{P}\left(\varphi, 2 l b_{1} ; \omega, L_{2} ; \psi, b_{1}\right)
$$

Step 3. We now show that (B4) in Lemma 3.1 is satisfied. If $u \in \bar{P}\left(\varphi, r_{1} ; \omega, L_{1}\right)$, by condition (A5), in the same way as in Step 1, we can obtain that $T: \bar{P}\left(\varphi, r_{1} ; \omega, L_{1}\right) \rightarrow P\left(\varphi, r_{1} ; \omega, L_{1}\right)$. Hence, condition (B4) in Lemma 3.1 is satisfied.

Step 4. Finally, we verify condition (B5) in Lemma 3.1 for $u \in \bar{P}\left(\varphi, r_{2} ; \omega, L_{2} ; \psi, b\right)$ with $\varphi(T u)>2 l b_{1}$. Then, by Lemma 2.2 and condition (A4), we have

$$
\begin{aligned}
\psi(T u) & =\min _{t \in\left[\frac{1}{l}, 1-\frac{1}{l}\right]_{\mathbb{T}}}|T u(t)| \\
& \geq \frac{1}{l} \max _{t \in[0,1]_{\mathbb{T}}}|T u(t)|-\frac{1}{l} \sum_{k=1}^{n}\left|\Delta(T u)\left(t_{k}\right)\right| \\
& \geq \frac{1}{l} \max _{t \in[0,1]_{\mathbb{T}}}|T u(t)|-\frac{1}{l} \sum_{k=1}^{n}\left|I_{k}\left(u\left(t_{k}\right)\right)\right|
\end{aligned}
$$




$$
\begin{aligned}
& >\frac{1}{l} 2 l b_{1}-\frac{1}{l} \frac{l b_{1}}{n} n \\
& =b_{1} .
\end{aligned}
$$

Thus, condition (B5) in Lemma 3.1 is satisfied.

Consequently, from Lemma 3.1, boundary value problem (1.1) has at least three positive solutions $u_{1}, u_{2}, u_{3} \in \bar{P}\left(\varphi, r_{2} ; \omega, L_{2}\right)$ with

$$
\begin{aligned}
& u_{1} \in P\left(\varphi, r_{1} ; \omega, L_{1}\right), \quad u_{2} \in\left\{\bar{P}\left(\varphi, r_{2} ; \omega, L_{2} ; \psi, b_{1}\right): \psi(u)>b_{1}\right\} \\
& u_{3} \in \bar{P}\left(\varphi, r_{2} ; \omega, L_{2}\right) \backslash\left(\bar{P}\left(\varphi, r_{2} ; \omega, L_{2} ; \psi, b_{1}\right) \cup \bar{P}\left(\varphi, r_{1} ; \omega, L_{1}\right)\right) .
\end{aligned}
$$

The proof is complete.

From the proof of Theorem 3.1, it is easy to see that if conditions like (A5)-(A7) are appropriately combined, we can obtain an arbitrary number of positive solutions of problem (1.1).

Corollary 3.1 Assume that (A1)-(A4) hold. There exist constants $0<r_{1}<b_{1}<2 l b_{1} \leq r_{2}<$ $b_{2}<2 l b_{2} \leq \cdots \leq r_{i}, 0<L_{1} \leq L_{2} \leq \cdots \leq L_{i}, i \in \mathbb{N}$, and the following conditions are satisfied:

(A8) $\max \left\{f(t, u, v), \bar{I}_{k}(u, v)\right\}<\min \left\{\phi_{p}\left(\frac{r_{h}}{H}\right), \phi_{p}\left(\frac{L_{h}}{L}\right)\right\}, I_{k}(u) \leq \frac{r_{h}}{H}$ for

$(t, u, v) \in[0,1]_{\mathbb{T}} \times\left[0, r_{h}\right] \times\left[0, L_{h}\right], 1 \leq h \leq i, 1 \leq k \leq n ;$

(A9) $f(t, u, v)>\phi_{p}\left(\frac{l b_{h}}{N}\right)$ for $(t, u, v) \in\left[\frac{1}{l}, 1-\frac{1}{l}\right]_{\mathbb{T}} \times\left[b_{h}, 2 l b_{h}\right] \times\left[0, L_{h+1}\right], 1 \leq h \leq i-1$.

Then problem (1.1) possesses at least $2 i-1$ positive solutions.

\section{An example}

Example 4.1 Let $\mathbb{T}=\left\{2^{-n}\right\}_{n \in \mathbb{N}^{2}} \cup\{0\}$. We consider the boundary value problem

$$
\left\{\begin{array}{l}
\left(\phi_{5 / 2}\left(u^{\Delta}(t)\right)\right)^{\nabla}+f\left(t, u(t), u^{\Delta}(t)\right)=0, \quad t \neq \frac{1}{4}, t \in[0,1]_{\mathbb{T}} \\
\Delta u\left(\frac{1}{4}\right)=-I_{1}\left(u\left(\frac{1}{4}\right)\right), \\
\Delta \phi_{5 / 2}\left(u^{\Delta}\left(\frac{1}{4}\right)\right)=-\bar{I}_{1}\left(u\left(\frac{1}{4}\right), u^{\Delta}\left(\frac{1}{4}\right)\right), \\
\phi_{5 / 2}\left(u^{\Delta}(0)\right)=\frac{1}{5} \phi_{5 / 2}\left(u^{\Delta}\left(\frac{1}{8}\right)\right), \quad u(1)=\frac{1}{10} u\left(\frac{1}{2}\right)
\end{array}\right.
$$

where

$$
\begin{aligned}
& f(t, u, v)= \begin{cases}\frac{1}{16} t^{2}+60\left(\frac{u}{2}\right)^{11}+\frac{1}{2}\left(\frac{v}{100}\right)^{4}, & u<2, \\
\frac{1}{16} t^{2}+60+\frac{1}{2}\left(\frac{v}{100}\right)^{4}, & u \geq 2,\end{cases} \\
& I(u)= \begin{cases}\frac{1}{16} u, & 0 \leq u \leq 1, \\
\frac{1}{16}, & u>1,\end{cases} \\
& \bar{I}(u, v)=\frac{1}{100} u+\frac{1}{200} v, \quad u \geq 0, v \leq 0 .
\end{aligned}
$$

Here $q(t) \equiv 1, p=\frac{5}{2}, n=1, m=3, t_{1}=\frac{1}{4}, \alpha_{1}=\frac{1}{5}, \beta_{1}=\frac{1}{10}, \xi_{1}=\frac{1}{8}, \eta_{1}=\frac{1}{2}$.

Choose $l=5, r_{1}=1, b_{1}=2, r_{2}=49, L_{1}=1, L_{2}=100$.

Then we have

$$
H=3.15779, \quad L=1.84201, \quad N=\left(\frac{3}{5}\right)^{2 / 3} .
$$


It is easy to verify that (A1)-(A4) hold and $f(t, u, v)$ satisfies

$$
\begin{aligned}
& \max \left\{f(t, u, v), \bar{I}_{1}(u, v)\right\} \leq 0.015<\min \left\{\phi_{5 / 2}\left(\frac{r_{1}}{H}\right), \phi_{5 / 2}\left(\frac{L_{1}}{L}\right)\right\} \approx 0.178207 ; \\
& I_{1}(u) \leq 0.0625<\frac{r_{1}}{H}=0.316677 \text { for }(t, u, v) \in[0,1]_{\mathbb{T}} \times[0,1] \times[0,1] ; \\
& f(t, u, v)>60>\phi_{5 / 2}\left(\frac{l b_{1}}{N}\right)=52.705 \text { for }(t, u, v) \in\left[\frac{1}{5}, \frac{4}{5}\right]_{\mathbb{T}} \times[2,20] \times[0,100] ; \\
& \max \left\{f(t, u, v), \bar{I}_{1}(u, v)\right\}<60.5625<\min \left\{\phi_{5 / 2}\left(\frac{r_{2}}{H}\right), \phi_{5 / 2}\left(\frac{L_{2}}{L}\right)\right\} \approx 61.125 ; \\
& I(u) \leq \frac{1}{16}<\frac{r_{2}}{H}=15.517 \quad \text { for }(t, u, v) \in[0,1]_{\mathbb{T}} \times[0,49] \times[0,100] .
\end{aligned}
$$

Thus, all the conditions of Theorem 3.1 hold. By Theorem 3.1, problem (4.1) has at least three positive solutions $u_{1}, u_{2}$, and $u_{3}$ such that

$$
\begin{aligned}
& \max _{t \in[0,1]_{\mathbb{T}}} u_{1}(t)<1, \quad \max _{t \in[0,1]_{\mathbb{T}}}\left|u_{1}^{\Delta}(t)\right|<1 ; \\
& 2<\min _{t \in\left[\frac{1}{5}, \frac{4}{5}\right]_{\mathbb{T}}} u_{2}(t) \leq \max _{t \in[0,1]_{\mathbb{T}}} u_{2}(t) \leq 49, \quad \max _{t \in[0,1]_{\mathbb{T}}}\left|u_{2}^{\Delta}(t)\right| \leq 100 ; \\
& 1<\max _{t \in[0,1]_{\mathbb{T}}} u_{3}(t) \leq 49, \quad \min _{t \in\left[\frac{1}{5}, \frac{4}{5}\right]_{\mathbb{T}}} u_{3}(t)<2, \quad \max _{t \in[0,1]_{\mathbb{T}}}\left|u_{3}^{\Delta}\right| \leq 100 .
\end{aligned}
$$

\section{Competing interests}

The authors declare that they have no competing interests.

\section{Authors' contributions}

All authors contributed equally in this article. They read and approved the final manuscript.

\section{Acknowledgements}

We would like to thank the reviewers for their valuable comments and suggestions on the manuscript.

\section{Received: 26 March 2013 Accepted: 25 September 2013 Published: 20 Nov 2013}

\section{References}

1. Lakshmikantham, V, Bainov, DD, Simeonov, PS: Theory of Impulsive Differential Equations. World Scientific, Singapore (1989)

2. Samoilenko, AM, Perestyuk, NA: Impulsive Differential Equations. World Scientific, Singapore (1995)

3. Liang, S, Zhang, J: The existence of countably many positive solutions for some nonlinear singular three-point impulsive boundary value problems. Nonlinear Anal. 71, 4588-4597 (2009)

4. LV, ZW, Liang, J, Xiao, TJ: Multiple positive solutions for second order impulsive boundary value problems in Banach spaces. Electron. J. Qual. Theory Differ. Equ. 38, 1-15 (2010)

5. Hilger, S: Analysis on measure chains - a unified approach to continuous and discrete calculus. Results Math. 18 18-56 (1990)

6. Bohner, M, Peterson, A: Dynamic Equations on Time Scales: An Introduction with Applications. Birkhäuser, Boston (2001)

7. Bohner, M, Peterson, A: Advances in Dynamic Equations on Time Scales. Birkhäuser, Boston (2003)

8. Feng, M, Feng, H, Zhang, $X$, Ge, W: Triple positive solutions for a class of $m$-point dynamic equations on time scales with p-Laplacian. Math. Comput. Model. 48, 1213-1226 (2008)

9. Karaca, IY, Tokmak, F: Existence of three positive solutions for $m$-point time scale boundary value problems on infinite intervals. Dyn. Syst. Appl. 20, 355-367 (2011)

10. Sun, HR, Li, WT: Existence theory for positive solutions to one-dimensional $p$-Laplacian boundary value problems on time scales. J. Differ. Equ. 240, 217-248 (2007)

11. Chen, H, Wang, H, Zhang, Q, Zhou, T: Double positive solutions of boundary value problems for $p$-Laplacian impulsive functional dynamic equations on time scales. Comput. Math. Appl. 53, 1473-1480 (2007)

12. Chen, $H$, Wang, $H$ : Triple positive solutions of boundary value problems for $p$-Laplacian impulsive dynamic equations on time scales. Math. Comput. Model. 47, 917-924 (2008)

13. Liang, $\mathrm{R}$, Shen, J: Triple positive solutions to bvp for $p$-Laplacian impulsive dynamic equations on time scales. Dyn. Contin. Discrete Impuls. Syst., Ser. A Math. Anal. 18, 719-730 (2011) 
14. Yin, L, Zhang, Z: Existence of a positive solution for a first-order $p$-Laplacian boundary value problems with impulsive on time scales. J. Appl. Anal. Comput. 2, 103-109 (2012)

15. Li, $\mathrm{P}, \mathrm{Chen}, \mathrm{H}, \mathrm{Wu}, \mathrm{Y}$ : Multiple positive solutions of $n$-point boundary value problems for $p$-Laplacian impulsive dynamic equations on time scales. Comput. Math. Appl. 60, 2572-2582 (2010)

16. Bai, Z, Ge, W: Existence of three positive solutions for some second-order boundary value problems. Comput. Math. Appl. 48, 699-707 (2004)

10.1186/1687-1847-2013-334

Cite this article as: Ozen et al.: Existence results for $p$-Laplacian boundary value problems of impulsive dynamic equations on time scales. Advances in Difference Equations 2013, 2013:334

Submit your manuscript to a SpringerOpen ${ }^{\odot}$ journal and benefit from:

- Convenient online submission

- Rigorous peer review

- Immediate publication on acceptance

- Open access: articles freely available online

- High visibility within the field

- Retaining the copyright to your article 